\title{
Clinicohematological Study of Pancytopenia in a Tertiary Care Hospital of Western Region of Nepal
}

\author{
Dilasma Ghartimagar, ' Arnab Ghosh,' Sushma Thapa,, Deepa Sapkota, 'Adarsh Kumar Jhunjhunwala, \\ Raghavan Narasimhan, 'OP Talwar' \\ 'Department of Pathology, Manipal College of Medical Science, Pokhara, Nepal.
}

\section{ABSTRACT}

Introduction: Pancytopenia is a relatively common hematological entity and is a manifestation of many illnesses which can be life threatening at times. The severity of pancytopenia and the underlying pathology determine the management and prognosis. This study was conducted to evaluate hematological and bone marrow findings in patients presenting with pancytopenia.

Methods: A prospective observational study was conducted in Department of Pathology, Manipal College of Medical Sciences, Pokhara from January 2011 to December 2016. Clinical and hematological parameters including bone marrow aspiration and biopsy were evaluated in all patients who presented with pancytopenia.

Results: Among 138 cases studied, patients' age ranged from 2 to 82 years with a mean age of 43.95 years, and there was male predominance. Most of the patients presented with generalized weakness, pallor, dypnoea and fever. Hypoplastic marrow was seen in 38 (27.5\%) cases, followed by $26(18.8 \%)$ cases of megaloblastic anemia and $19(13.76 \%)$ cases of acute leukemia. Other findings included one case each of hemophagocyosis, leishmaniasis, plasmodium vivex malaria and metastatic carcinoma.

Conclusions: This study highlights that pancytopenia is a common hematological problem and that the study of detailed primary hematological investigations along with bone marrow study in patients with pancytopenia will help to identify the cause for further planning and management.

Keywords: hypoplastic marrow; leukemia; megaloblastic anemia; pancytopenia.

\section{INTRODUCTION}

Pancytopenia is an important clinico-hematological condition which is characterized by reduction of all major cell lines i.e. red cells, white cells and platelets in peripheral blood resulting in anemia, thrombocytopenia and leucopenia leading to symptoms of generalized weakness, pallor, bleeding manifestation and susceptibility to different infections. ${ }^{1}$

Pancytopenia in an adult is called when hemoglobin level is less than $13.3 \mathrm{gm} / \mathrm{dl}$ in males or $11.5 \mathrm{gm} / \mathrm{dl}$ in females, the leukocyte count is less than $4000 / \mathrm{mm}^{3}$, and the platelet count is less than $150,000 \mathrm{~mm} .^{2}$ Different mechanisms lead to development of pancytopenia like decreased production of hematopoietic cells, ineffective hematopoiesis, and bone marrow infiltration by cancer

Correspondence: Dr. Dilasma Ghartimagar, Department of Pathology, Manipal College of Medical Science, Pokhara, Nepal. Email: dilasmagm@ @otmail.com, Phone: +977- 9840603962. 
cells. ${ }^{3}$

This study was done to identify different underlying causes of pancytopenia with the help of detailed hematological and bone marrow findings in our set up and finally to help the patients with proper treatment and management.

\section{METHODS}

A prospective observational study was conducted in the Department of Pathology, Manipal College of Medical Sciences, Pokhara from January 2011 to December 2016. Ethical approval was taken from MEMG/IRC/GA. Patients of all age groups and both genders were included. Case selection was based on the clinical features which were mainly supported by laboratory evidence of pancytopenia. Clinical details of pancytopenia with regard to drug intake, generalized weakness, anorexia, fever, weight loss, bleeding tendency, organomegaly, lymphadenopathy and bone pain were recorded. Patients receiving chemotherapy for malignancy or who had received recent blood or platelet transfusions were excluded from the study.

Hematological investigations such as complete blood count (CBC) were analyzed by Automated Hematology Analyzer LabLife H3D Premier with blood sample taken in EDTA tube. Pancytopenia was diagnosed in presence of anemia (hemoglobin level less than 13.3 $\mathrm{gm} / \mathrm{dl}$ in males or $11.5 \mathrm{gm} / \mathrm{dl}$ in females), leucopenia (total leukocyte count [TLC] $<4,000 / \mathrm{mm}^{3}$ ) and thrombocytopenia (platelet count $<150,000 / \mathrm{mm}^{3}$ ). The peripheral smears were stained with Leishman stain and Supravital stain was used for reticulocyte count. Platelet counts were further verified manually. For bone marrow examination, the procedure was explained to the patient and informed consent was taken in all cases. Bone marrow aspiration was done in a conventional manner. Bone marrow trephine biopsies were performed in all the adult cases except in pediatric age group. Bone marrow aspiration smears were stained by Leishman stain and examined in detail. Trephine biopsy sections were stained with Hematoxylin and Eosin. Special stains like Iron stain, Myeloperoxidase stain, Sudan black, Periodic Acid Schiff and Masson's Trichrome stain were done whenever required. The data were analyzed using Microsoft Excel and SPSS 21.0 version.

\section{RESULTS}

During the study period, 302 bone marrow examinations were done and a total of 138 patients presented with pancytopenia. There were 85 male and 53 female patients with a male to female ratio of $1.6: 1$. The age of patients ranged from 2 to 82 years with a mean age of 43.95 years and maximum number of cases $23(16.6 \%)$ were seen in three different age groups (Table1). Patients presented with various complaints like generalized weakness, pallor, dyspnea, fever, organomegaly, weight loss, etc (Table 2). Generalized weakness and pallor was noted in most of the cases. Splenomegaly and hepatomegaly were seen in cases of megaloblastic anemia, acute leukemia and normal marrow. Bony tenderness was seen in one case each of acute leukemia and megaloblastic anemia. Lymphadenopathy along with anemia and organomegaly was noted in 11 years old female of acute lymphoblastic leukemia.

\begin{tabular}{|ccccc|}
\hline \multicolumn{5}{|c|}{ Table 1. Age wise distribution of pancytopenia cases. } \\
\hline $\begin{array}{c}\text { Age group } \\
\text { (yrs) }\end{array}$ & Male & Female & $\mathbf{n}(\%)$ & $\mathbf{C l}$ \\
$<10$ & 7 & 3 & $10(7.2)$ & $(3.90,8.29)$ \\
$11-20$ & 13 & 10 & $23(16.6)$ & $(14.70,16.94)$ \\
$21-30$ & 7 & 16 & $23(16)$ & $(24.26,27.20)$ \\
$31-40$ & 7 & 2 & $9(6.5)$ & $(33.24,37.86)$ \\
$41-50$ & 3 & 3 & $6(4.3)$ & $(37.28,47.82)$ \\
$51-60$ & 19 & 4 & $23(16.6)$ & $(57.04,58.40)$ \\
$61-70$ & 13 & 9 & $22(15.9)$ & $(64.47,67.20)$ \\
$71-80$ & 14 & 6 & $20(14.49)$ & $(74.89-77.90)$ \\
$>80$ & 2 & - & $2(1.44)$ & $(80.52-82.48)$ \\
Total & 85 & 53 & $138(100)$ \\
\hline
\end{tabular}

Table 2. Presenting complaints and clinical findings.

\begin{tabular}{|ll|}
\hline Colinical findings & $\mathbf{n}(\%)$ \\
Generalized weakness & $120(86.95)$ \\
Pallor & $93(67.39)$ \\
Dyspnoea & $55(42.30)$ \\
Fever & $45(32.60)$ \\
Splenomegaly & $27(19.56)$ \\
Hepatomegaly & $15(10.86)$ \\
Bleeding manifestation & $18(13.04)$ \\
Easy bruising & $1(0.72)$ \\
Weight loss & $5(3.6)$ \\
Decrease appetite & $8(5.79)$ \\
HTN & $4(2.89)$ \\
Lymphadenopathy & $3(2.17)$ \\
Myalgia & $3(2.17)$ \\
Bony tenderness & $2(1.44)$ \\
Jaundice & $1(0.72)$ \\
COPD & $1(0.72)$ \\
Incomplete abortion & $1(0.72)$ \\
\hline
\end{tabular}

Hematological parameters of different causes of 
pancytopenia showed different values. Hemoglobin level was as low as $2.1 \mathrm{gm} / \mathrm{dl}$ in case of dimorphic anemia, TLC as low as $900 / \mathrm{cmm}$ in megaloblastic anemia and platelets as low as $1000 / \mathrm{cmm}$ in hypoplastic marrow (Table 3).

Peripheral smear showed dimorphic anemia in 52 $(37.68 \%)$ cases, normocytic normochromic anemia in $47(34.05 \%)$ cases and microcytic hypochromic anemia in $23(16.66 \%)$ cases. Macrocytic anemia in
$16(11.59 \%)$ cases showed macro-ovalocytes and hypersegmented neutrophils in a peripheral smear. Leucopenia and thrombocytopenia were seen in all cases.

In bone marrow study, the most common cause of pancytopenia was hypoplastic marrow which was seen in 38 (27.53\%) cases, followed by megaloblastic anemia seen in $26(18.8 \%)$ cases and acute leukemia seen in $19(13.76 \%)$ cases (Table 4$)$.

Table 3. Hematological parameters of pancytopenia cases.

\begin{tabular}{llll}
\hline BM Diagnosis & Hb (gm/dl) & TLC (/cumm) & Platelets (/cumm) \\
Hypoplastic marrow & $2.9-10.9$ & $1,500-3,800$ & $1000-100,000$ \\
Megaloblastic Anemia & $2.9-12.2$ & $900-3,600$ & $4000-110,000$ \\
Dimorphic Anemia & $2.1-10.7$ & $2600-3800$ & $14,000-116,000$ \\
Acute Leukemia AML & $3-10.6$ & $100-3700$ & $11,000-7,2000$ \\
Acute Leukemia ALL & $4.3-6$ & $2100-3975$ & $23000-97,000$ \\
Lymphoma & $3.6-4.5$ & $1000-3500$ & $30,000-39,000$ \\
MDS & $3.8-8.9$ & $1500-3600$ & $12,000-99,000$ \\
Erythroid Hyperplasia & $4.4-10$ & $1200-3600$ & $32,000-98,000$ \\
Normal marrow & $6.6-9.9$ & $2100-3800$ & $22,000-100000$ \\
Diluted marrow & $3.1-10$ & $1300-3800$ & $24,000-80,000$ \\
Hemophagocytosis & 10.5 & 3500 & 27,000 \\
LD body & 8 & 1300 & 40,000 \\
P. vivex malaria & 9.1 & 3600 & 89,000 \\
Carcinoma Mets & 5.6 & 2000 & 60,000 \\
Others & $5.6-8.9$ & $900-2400$ & $29,00-72,000$ \\
\hline
\end{tabular}

Hypoplastic marrow was seen in 38 cases and there were 23 males and 15 females with a male to female ratio of 1.5:1. The mean age was 48.07 years with a range from 10 to 80 years. There was a distinct male preponderance among all the groups especially above the age of 40 years. Peripheral smear showed normocytic normochromic to microcytic hypochromic RBC picture. Macrocytes were seen in four cases of hypoplastic marrow. Bone marrow study showed hypocellularity with suppression of all erythroid, myeloid and megakaryocytic cell lineages with a relative lymphocytosis. Two cases of hypoplastic marrow showed aplastic anemia in trephine biopsy.
Megaloblastic anemia was observed in 26 cases. There were 20 males and 6 females with male to female ratio of 3.3:1 in megaloblastic anemia. The age ranged from 5 to 82 years with mean age of 43.46 years. Macro-ovalocytes and hypersegement neurtrophils were seen in the peripheral smear study. Bone marrow aspiration showed megaloblastic erythroid hyperplasia. Megaloblasts had the characteristic feature of nucleus showing sieve like nuclear chromatin, nuclear cytoplasmic asynchrony in maturation with some of the cells showing cytoplasmic blebs. Giant metamyelocytes and band forms were predominant in granulocytic series. 


\begin{tabular}{|ll|}
\hline \multicolumn{2}{|l|}{ Table 4. Bone marrow diagnosis of cases in } \\
Pancytopenia. & $\mathbf{n}(\%)$ \\
\hline Bone Marrow Diagnosis & $38(27.5)$ \\
Hypoplastic marrow & $26(18.8)$ \\
Megaloblastic Anemia & $7(5.0)$ \\
Dimorphic Anemia & $16(11.6)$ \\
Acute Leukemia - AML & $3(2.2)$ \\
Acute Leukemia - ALL & $2(1.4)$ \\
Lymphoma & $11(7.9)$ \\
MDS & $10(7.2)$ \\
Erythroid Hyperplasia & $10(7.2)$ \\
Normal marrow & $7(5.0)$ \\
Diluted marrow & $1(0.7)$ \\
Hemophagocytosis & $1(0.7)$ \\
LD body & $1(0.7)$ \\
P. vivex malaria & $1(0.7)$ \\
Metastatic Carcinoma & $4(2.8)$ \\
Others & $138(100)$ \\
Total &
\end{tabular}

We had 19 cases of acute leukemia, which comprises of 16 cases of acute myeloid leukemia (AML) and 3 cases of acute lymphoblastic leukemia (ALL). The age range for acute leukemia was 2 to 78 years. ALL was seen mainly in pediatric age group of 2 to 17 years with a mean age of 8 years. Here, most of the patients presented with pallor and fever; there was only one case who presented with bony tenderness. Clinically, all the patients were with a provisional diagnosis of anemia/pancytopenia under evaluation. Bone marrow picture showed hypercellular marrow in 17 cases and hypoplastic marrow in two cases of acute leukemia. Erythroid and megakaryocytic series were markedly suppressed in all cases of acute leukemia who presented as pancytopenia. Majority of cells were myeloblasts and lymphoblasts, constituting more than $40 \%$ and $30 \%$ of cells in the marrow, respectively. Bone marrow aspiration showed myeloblasts with Auer rods along with Faggot cells in two cases of AML- M3.

There were two cases of hypoplastic marrow due to lymphoma infiltration. One case was of 58 years old male with bone marrow infiltration by Hodgkin Lymphoma and another case was of 60 years old female with marrow infiltration by Non Hodgkin Lymphoma.

Myelodysplastic syndrome (MDS) presenting with pancytopenia was observed in 11 cases. Refractory anemia with multilineage dysplasia was seen in three cases and one case was of refractory anemia. The remaining seven cases were reported as MDS and further sub typing was not done. The age range for
MDS was 30 to 79 years. There was only one case of male with 30 years of age while the rest were above 60 years. Erythroid hyperplasia and normal marrow were noted in 10 cases each of patients who presented with pancytopenia. Diluted marrow was seen in seven cases which was mainly noted in pediatric age group. There was one case of 15 years old female with a history of heavy menstrual bleeding and loss of consciousness who also presented with pancytopenia. Her bone marrow picture showed erythroid hyperplasia.

There was also one case of hemophagcytosis in 61 years old female who presented with fever for 3 months, pallor and splenomegaly. Her bone marrow picture showed increased histiocytes with emperipolisis. On follow up, the patient had died three months after the bone marrow diagnosis of hemophagocytosis. One 16 years old male patient was diagnosed as Leishmaniasis. His bone marrow picture showed LD bodies. Another 60yrs old female presented with pancytopenia but the bone marrow aspiration showed schizonts of plasmodium vivex malaria. Her peripheral smear picture also showed few ring forms and schizonts of plasmodium vivex malaria. One 71 years old male presented severe pancytopenia and his bone marrow picture showed metastatic adenocarcinoma deposits. Clusters of tumor cells were present with marked suppression of the erythroid, myeloid and megakaryocytic series of cells.

\section{DISCUSSION}

Pancytopenia itself is not a disease but many serious and life threatening conditions can manifest as pancytopenia. It has different etiologies, with a variation in frequency of different diseases leading to pancytopenia in different population groups. ${ }^{3}$ The different causes of pancytopenia may be attributed to multitude of various factors such as geographical variations, nutrition, prevalence of certain infections and exposure to different toxic chemicals or pesticides in a particular population under study..$^{4,5}$

Pancytopenia is usually suspected on clinical grounds when a patient presents with unexplained generalized weakness, pallor, prolonged fever and organomegaly. In the present study, the most common presenting symptom was generalized weakness which was seen in $120(86.95 \%)$ cases followed by pallor and dyspnoea which were seen in $95(67.39 \%)$ cases and 55 $(42.30 \%)$ cases respectively. In other studies also, the most common presenting complaint was generalized weakness, pallor and dyspnea. . $^{3,4,6}$

In the present study of 138 patients, definite male predominance was observed with a M:F ratio of 1.6:1 and a mean age of 43.95 years. In a similar study done by Gayathri et al, with 104 patients showed male to 
female ratio of $1.2: 1$ and mean age was 41 years. ${ }^{6}$ In another study done by Mir et al, with 138 cases also showed male predominance with a M:F ratio of $1.3: 1$ and a mean age of 43.81 years. ${ }^{3}$ Bone marrow examination is a frequently asked investigation to know the cause pancytopenia as in our set up. ${ }^{7}$

The most common cause of pancytopenia reported from various studies worldwide has been hypoplastic marrow. ${ }^{8}$ Incidence of hypoplastic anemia varies from $10 \%$ to $52 \%$ among pancytopenic patients. ${ }^{6}$ In our study also, the most common cause of pancytopenia was hypoplastic marrow which was observed in 38 cases. There were two cases of aplastic anemia which were diagnosed on trephine biopsy. In studies, done by Pathak et al, and Lakhey et al, of Nepal also showed hypoplastic marrow as the commonest cause of pancytopenia.9,10 But Vaidya $S$ in his study from Nepal showed the commonest cause of pancytopenia as megaloblastic anemia. ${ }^{7}$ Khodke et al, also found megaloblastic anemia as the most common cause for pancytopenia in his study of 250 cases from India. ${ }^{11}$

Different studies showed different causes of Pancytopenia. According to the study done by Mir et al, $^{3}$ megaloblastic anaemia was $72.7 \%$ and acute leukaemia $6.8 \%$, by Rehmani et al, ${ }^{4} 20 \%$ megaloblastic anemia and $27.05 \%$ aplastic anemia, by Gayathri et al, ${ }^{6}$ Megaloblastic Anaemia 74\% and Aplastic Anaemia $18 \%$, by Vaiday S, ${ }^{7}$ Megaloblastic anemia $34.95 \%$,

Aplastic anemia $31.33 \%$, by Pereira ADS et al, ${ }^{8}$ Megaloblastic anemia (60\%), Aplastic anemia and leukemia (10\% each), by R Pathak et al, ${ }^{9}$ Hypoplastic anemia (42.15\%) and Hematological malignancies (19.4\%), by Lakhey et al, ${ }^{10}$ Hypoplastic anemia (29.6\%) and Hematological malignancies $(27.78 \%)$, by Khodke $\mathrm{K}$ et al, ${ }^{11}$ Megaloblastic anaemia (44) and Aplastic anaemia (14), by Graham $S$ et al, ${ }^{13}$ Normoblastic erythroid hyperplasia (30\%), Megaloblastic anemia (20\%), by Jha et al, ${ }^{14}$ Hypoplastic anemia $(29.05 \%)$ and Megaloblastic anemia (23.64\%).

Present study shows Hypoplastic anemia (27.5\%) and Megaloblastic anemia (18.8\%).

The second most common cause of pancytopenia in the present study was megaloblastic anemia, accounting for $26(18.8 \%)$ cases. But Gyathri et al, and Pereira et al, found megaloblastic anemia as the most common cause of pancytopenia in their studies and incidence was $60 \%$ and $74.04 \%$ respectively. ${ }^{6,8}$

The incidence of acute leukemia cases for the cause of pancytopenia varies between $1.61 \%-14.5 \%$ in different studies. ${ }^{3}$ In the present study, acute leukemia is the third most common cause for pancytopenia. We found that 19 cases of acute leukemia where 16 (13.8\%) cases were of AML and 3 cases were of ALL. Lakhey et al, had 15 (27.78\%) cases and Mir et al, had 9 (6.78\%) cases in their studies and both found acute leukemia as the second most common cause of pancytopenia. ${ }^{3,10}$ General population showed the occurrence of MDS as 5 per 1,00,000 people. However, in older individuals of age more than 70 years, the incidence increases from 22-45 per 1,00,000 people and increasing further with the age. ${ }^{10}$ Present study showed $11(7.9 \%)$ cases of MDS. Rehamani et al, found 4 cases of MDS out of 244 patients in four years' study and Lakhey et al, also found $4(7.4 \%)$ cases of MDS in his study. ${ }^{4,10}$ Shah et al, encountered with only $1(2.5 \%)$ case of MDS in their study of 40 cases of pancytopenia. ${ }^{12}$

Erythroid hyperplasia and normal marrow were seen in 10 cases each in the present study. Graham et al, found erythroid hyperplasia in $9(30 \%)$ cases and it was the most common cause of pancytopenia in their study of 30 cases who presented as pancytopenia. ${ }^{13}$ Lakhey et al, and Jha et al, also found $6(11.11 \%)$ cases and $29(19.6 \%)$ cases of erythroid hyperplasia in their study of pancytopenia. ${ }^{10,14}$ Same studies from Nepal also showed $4(7.41 \%)$ cases and $5(3.38 \%)$ cases of normal marrow in their studies. ${ }^{10,14}$

We had 1 case of hemophagocytosis in 61 years old female who presented as pancytopenia. Pereira ADS et al, also encountered with only one case of hemophagocytosis in their study of 80 cases. $^{8}$

We also encountered with $1(0.7 \%)$ case of Leishmaniasis in the present study. Rehmani et al, and Jha et al, also had 1 case each of Leishmaniasis in their study. ${ }^{4,14}$

We had 1 case of malaria in our study, constituting $0.7 \%$ of total cases whereas Pereira ADS et al, and Gayathri $\mathrm{BN}$ et al, have reported an incidence of $1(2.5 \%)$ case and $2(1.93 \%)$ cases of malaria respectively in their studies. 6,8

The present study also had $1(0.7 \%)$ case of 71 years old male diagnosed as metastatic adenocarcinoma who presented as pancytopenia. The primary tumor was adenocarcinoma of the right lung. Jha $A$ et al, also had $1(0.67 \%)$ case of metastatic neuroblastoma in their study of 148 cases from Nepal. ${ }^{14}$ Vaidya S also had only $1(1.20 \%)$ case of metastatic carcinoma in his study of 83 cases from Nepal. ${ }^{7}$

The limitation of the study is that it is a small sample size and single centered. Larger sample size and proper sampling would have provided a clearer picture of the situation. 


\section{CONCLUSIONS}

Pancytopenia is an important clinico-hematological condition and our study showed that hypoplastic marrow was the commonest cause of pancytopenia followed by megaloblastic anemia and acute leukemia.
Clinical assessment followed by a complete hemogram and bone marrow study is an essential step in planning, further management and prognosis of pancytopenia cases.

\section{Conflict of Interest: None.}

\section{REFERENCES}

1. Tariq M, Khan N, Basri R, Amin S. Aetiology of Pancytopenia. Professional Med J. 2010;17:252-6. [Full Text]

2. Khunger JM, Arulselvi S, Sharma U, Ranga S, Talib VH. Pancytopenia - A Clinico Haematological Study of 200 Cases. Indian J Pathol Microbiol. 2002;45:375-9. [PubMed | Full Text]

3. Mir TA, Bhat MH, Raina AA. Etiological Profile of Pancytopenia in a Tertiary Care Hospital of Kashmir Valley. International Journal of Science and Research. 2015;10(4):1186-9. [ Full Text]

4. Rehmani TH, Arif M, Haider S, Arif S, Ahmad R, Saeed M. Spectrum of Pancytopenia; A tertiary care experience. Professional Med J. 2016;23(5):620-6. [Full Text]

5. Kumar R, Kalra SP, Kumar H, Anand AC. Madan H: Pancytopenia - A six year study. J Assoc Physicians India. 2001;49:1078-81. [PubMed]

6. Gayathri BN, Rao KS. Pancytopenia: A clinico hematological study. J Lab Physicians. 2011;3:15-20. [Full Text | DOI]

7. VaidyaS. Evaluation of bone marrow in cases of pancytopenia in a tertiary care hospital. Journal of Pathology of Nepal. 2015;5:691-5. [Full Text]
8. Pereira ADS, Dias A. Hematological Analysis of Pancytopenia: A Prospective Study. International Journal of Scientific Study. 2016;4(4):71-8. [피l Text | DOI]

9. Pathak R, Jha A, Sayami G. Evaluation of bone marrow in patients with pancytopenia. Journal of Pathology of Nepal. 2012;2:265-71. [Full Text]

10. Lakhey A, Talwar OP, Singh VK, KC Shiva Raj. Clinico-hematological study of pancytopenia. Journal of Pathology of Nepal. 2012;2:207-10. [DOI | Full Text]

11. Khodke K, Marwah S, Buxi G, Yadav RB, Chaturvedi NK. Bone Marrow Examination in Cases of Pancytopenia. Journal, Indian Academy of Clinical Medicine. 2001;2:55-9. [Full Text]

12. Shah P, Patel RD, Gamit B, Gheewala S. Bone marrow examination in cases of pancytopenia. Int J Res Med Sci. 2017;5(4):1494-8. [DOI [ Full Text]

13. Graham S, Marla NJ, Fernandes H, Jayaprakash CS. A clinicohematological evaluation of pancytopenia in a tertiary care hospital in South India. Muller Journal of Medical Sciences and Research. 2015;6(1):5-9. [ Full Text]

14. Jha A, Sayami G, Adhikari RC, Panta AD, Jha R. Bone Marrow Examination in Cases of Pancytopenia. J Nepal Med Assoc. 2008; 47(169):12-7. [PubMed ] 